\title{
La sindrome anemica cardio-renale. Terza parte: Terapia
}

\author{
Rodolfo Fernando Rivera', Maria Teresa Sciarrone Alibrandi², \\ Luca Di Lullo ${ }^{3}$, Fulvio Floccari ${ }^{4}$, Antonio De Pascalis ${ }^{5}$, \\ Antonio Bellassi ${ }^{6}$ e Claudio Ronco ${ }^{7}$
}

\section{Cardio-renal anemia syndrome. Third part: Therapy}

In the previous sections of this chapter the clinical, epidemiological, pathophysiological and diagnostic aspects of cardiorenal anemia syndrome (CRAS) were treated. In this third and final component of the review the updates on the therapeutic aspects will be addressed. Erythropoiesis-stimulating agents (ESAs) and adjuvant iron therapy represent the primary treatment for anemia in CRS. The latest randomized trials for the treatment of iron deficiency in CRAS using intravenous (i.v.) Fe, have shown an improvement in symptoms, functional capacity and quality of life. These beneficial effects were independent of the presence of anemia. Furthermore, treatment with i.v. Fe can reduce the hospitalization rate due to the worsening of CHF. Oral iron is available at a lower cost than i.v. Fe, but its use did not translate into beneficial effects in CHF patients with iron deficiency (ID). The use of ESAs has been recently debated; the latest interventional study seems to demonstrate a neutral or negative effect in the active arm with darbepoetin treatment. These findings contrast with previous single-blind studies and meta-analyses, which showed an improvement in quality of life, left ventricular systolic function, and exercise tolerance following ESA therapy. In this review we discuss interventional studies in patients with CRAS and the potential role of ESA in this setting.

\section{Keywords}

Cardiorenal anemia syndrome (CRAS), Chronic kidney disease (CKD), Congestive heart failure (CHF), iron therapy, erythropoiesis-stimulating agents, blood transfusions

\section{Introduzione}

Nei capitoli precedenti, ${ }^{1}$ si è puntualizzato sugli aspetti clinici ed epidemiologici, clinici e diagnostici della sindrome anemica-cardio renale (CRAS). ${ }^{2}$ Malattie croniche come lo scompenso cardiaco congestizio (CHF) e alla malattia renale cronica (CKD) coesistono con l'anemia aumentando sensibilmente il rischio di mortalità.

Nel corso degli ultimi decenni è stato possibile assistere ad un significativo sviluppo della terapia del paziente affetto da CHF. Le linee guida ESC $2016^{3}$ per la gestione della CHF raccomandano un percorso diagnostico e terapeutico basato sull'evidenza, volto a migliorare i sintomi e la prognosi della CHF. Tale percorso include l'uso appropriato di inibitori dell'enzima di conversione dell'angiotensina (ACE-i)/bloccanti dei recettori dell'angiotensina (ARB), beta-bloccanti, antagonisti dei recettori mineralcorticoidi,
'U.O. Nefrologia e Dialisi, Ospedale San Gerardo Hospital, ASST Monza, Monza, Italia

2U.O. Nefrologia, Dialisi e Ipertensione, IRCCS Ospedale San Raffaele, Milano, Italia

${ }^{3}$ U.O. Nefrologia e Dialisi, Ospedale L. Parodi - Delfino, Colleferro, Roma, Italia

${ }^{4}$ U.O. Nefrologia e Dialisi, Ospedale San Paolo, Civitavecchia, Italia ${ }^{5}$ U.O. Nefrologia e Dialisi, Ospedale V. Fazzi, ASL Lecce, Lecce, Italia 6U.O. Nefrologia e Dialisi, Ospedale Sant'Anna, ASST Lariana, Como, Italia

7U.O. Nefrologia e Dialisi, Ospedale San Bortolo \& International Renal Research Institute Vicenza (IRRIV), Vicenza, Italia

\section{Corrispondenza:}

Rodolfo F. Rivera, Division of Nephrology and Dialysis, San Gerardo Hospital, ASST Monza, Via Pergolesi 33, 20900 Monza, Italia.

E-mail: rodolfofrivera@gmail.com 
sacubitril/valsartan, ivabradina e la "device therapy" (defibrillatori cardioverter impiantabili [ICDs] e terapia di resincronizzazione cardiaca [CRT]) in tutti i pazienti con CHF e ridotta funzione sistolica (raccomandazioni I, II o classe IIa). ${ }^{3}$ Queste raccomandazioni terapeutiche esprimono i risultati di diversi trial multicentrici a larga scala. Tuttavia, la CHF, specialmente se associata ad altre comorbidità come la CKD e l'anemia, continua ad essere una malattia ad alta letalità e a crescente onere per i sistemi sociosanitari. Infatti, la forte associazione di anemia con il progressivo decadimento delle condizioni generali e gli alti tassi di mortalità ha portato ad una ricerca crescente che vede nel trattamento dell'anemia un bersaglio terapeutico nei pazienti con CHF.

Purtroppo, a causa dell'attuale discordanza nella classificazione e definizione dell'anemia in pazienti con CHF, ${ }^{1}$ gli studi interventistici condotti finora hanno riportato risultati diversi. Appare quindi sempre più essenziale la esigenza di chiarire alcuni importanti quesiti, quali:

$\checkmark$ I pazienti con CHF e anemia potrebbero trarre beneficio in termini di qualità della vita e/o di parametri funzionali dall'uso di agenti stimolanti l'eritropoiesi (erythropoiesis-stimulating agents, ESA)?

$\checkmark$ Si giustifica l'avvio di una terapia con ESA ignorando l'esistenza di carenza di ferro (iron deficit, ID)?

$\checkmark$ Sono da trattare tutte le forme di ID prima di iniziare la terapia ESA?

$\checkmark$ Quale via di somministrazione del ferro (Fe) favorire, di fronte al rischio clinico e al rapporto costo beneficio?

$\checkmark$ Quale target di mantenimento di $\mathrm{Hb}$ nei pazienti con CRAS per migliorare gli esiti?

Il presente capitolo riporta i risultati degli ultimi trial e i più importanti studi osservazionali e si propone di dare risposta ad alcuni dei precedenti questioni.

\section{Trattamento dell'anemia nella sindrome cardio- renale}

Il dibattito che caratterizza l'aspetto terapeutico dell'anemia nella SCR è l'effetto diretto di almeno due fattori: da una parte la capacità da parte del clinico ad adottare gli strumenti diagnostici finora descritti, e da un'altra un visibile dissenso nell'indicazione sull'inizio della terapia e dei farmaci da utilizzare. Mentre in ambito nefrologico, ${ }^{4-6}$ le linee guida internazionali appaiono molto esplicite per il trattamento dell'anemia, nel contesto cardiologico, ${ }^{3}$ vengono riportate considerazioni di carattere generali.

Gli schemi attuali propongono tre possibilità terapeutiche per aumentare l'Hb nei pazienti con CHF: la trasfusione di sangue, la supplementazione di Fe e la somministrazione di agenti stimolanti l'eritropoiesi (ESA).

In accordo con le linee guida americane, ${ }^{7}$ l'emotrasfusione è raccomandata per i livelli di $\mathrm{Hb}$ nell' intervallo da $6-8 \mathrm{~g} / \mathrm{dL}$. Il beneficio clinico dell'emotrasfusione per livelli più elevati non è ben stabilito. Sebbene la l'emotrasfusione è indicata nella terapia dell'anemia acuta, questo trattamento si associa a potenziali effetti collaterali come immunosoppressione, incremento del rischio di infezioni, sovraccarico di volume, sovraccarico di Fe e sensibilizzazione agli antigeni HLA. Inoltre questo approccio fornisce solo effetti temporanei.

Nel paziente cardiopatico cronico invece, l'approccio terapeutico è orientato alla supplementazione di $\mathrm{Fe}$ e all'uso di ESA. È necessario considerare, però, che i pochi studi disponibili in letteratura relativi all'anemia nella CRAS non sempre sono controllati o randomizzati e spesso sono dotati di casistiche limitate nel numero e nel tempo di osservazione. Queste caratteristiche potrebbero limitare la validità dell'evidenza ottenuta.

\section{La terapia con ferro nell'anemia cardio-renale}

Recentemente, sono stati condotti degli studi clinici controllati randomizzati (RCT) volti a studiare gli effetti del trattamento con $\mathrm{Fe}$ e.v. nei pazienti con CHF e ID, indipendentemente della presenza di anemia. I risultati confermano che l'astenia e la limitata capacità di esercizio misurata con il test del cammino, sono i sintomi principali della ID anche in assenza di anemia. In tale contesto, alcuni autori suggeriscono che l'integrazione di $\mathrm{Fe}$ migliora l'adattamento all'attività física aerobica ${ }^{8}$ e la capacità di resistenza. ${ }^{9}$ In aggiunta, lo studio PREFER (RCT) ha rilevato che una singola dose di ferro carbossimaltosio (FCM) riduceva rapidamente l'affaticamento e migliorava i punteggi delle funzioni cognitive ${ }^{10}$ in pazienti cardiopatici con ID ma senza anemia.

Un numero crescente di evidenze suggerisce che in pazienti con CHF, l'ID si associa a ridotta capacità di esercizio, ridotta qualità della vita e aumento della morbilità e della mortalità, anche in assenza di anemia conclamata. ${ }^{11}$ Dal punto di vista prognostico, l'alterazione dell'omeostasi del Fe ha una impronta più infausta della stessa anemia. Tra i pazienti cardiopatici cronici infatti, il rischio di morte si duplica tra quelli con ID senza anemia rispetto a quelli anemici con eccesso di Fe. ${ }^{12}$

Recentemente, le linee guida ESC 2016 per la gestione della $\mathrm{CHF}^{3}$ hanno definito un percorso terapeutico volto a migliorare i sintomi e la prognosi della CHF. Sebbene siano disponibili soltanto pochi e piccoli studi sulla terapia con Fe in pazienti anemici e CHF, le linee guida ESC 2016 raccomandano una ricerca di routine per l'ID determinando i livelli di ferritina e di saturazione della transferrina (TSAT) in tutti i pazienti con CHF (raccomandazione di classe I, livello C). Nel caso di anemia da ID, deve essere preso in considerazione un trattamento con FCM e.v. in 
pazienti CHF sintomatici, ambulatoriali con ridotta frazione di eiezione del ventricolo sinistro (FEVS) (classe IIa, raccomandazione di livello A) per alleviare i sintomi della $\mathrm{CHF}$ e migliorare la capacità di esercizio e la qualità della vita indipendentemente dalla presenza dell'anemia.

Nonostante le attuali raccomandazioni, gli sforzi clinici per diagnosticare e trattare l'ID nei pazienti con CHF rimangono bassi in gran parte dei paesi europei quando invece dovrebbero essere fortemente incoraggiati. ${ }^{13}$

Anche se la terapia con $\mathrm{Fe}$ potrebbe essere utile già nelle prime fasi della CHF, le attuali linee guida non forniscono precise indicazioni sulla fase della progressione della malattia in cui debbano essere avviate sia le indagini diagnostiche che l'impostazione terapeutica della ID.

Il Fe orale viene solitamente somministrato sotto forma di sali ferrosi,${ }^{14}$ come il solfato ferroso, che è ampiamente utilizzato e disponibile a un costo inferiore rispetto al $\mathrm{Fe}$ e.v. Tuttavia, è necessario puntualizzare che il suo assorbimento per via orale è generalmente scarso e circa il $60 \%$ dei pazienti presenta effetti collaterali, ${ }^{14}$ maggiormente di tipo gastrointestinali. Questo problema è più intensamente espresso in pazienti con $\mathrm{CHF},{ }^{15}$ probabilmente come conseguenza all'eccesso cronico di volume in grado di indurre edema parietale del tratto gastroenterico impedendo l'adeguato assorbimento, o semplicemente a causa dell'interazione con i numerosi farmaci a cui questi pazienti sono quotidianamente esposti. La dose media di Fe elemento necessaria per ottenere la replezione marziale in pazienti con CHF e ID è di $1000 \mathrm{mg}$, e di conseguenza il reintegro dei depositi marziali con la terapia orale richiederà un tempo di trattamento superiore ai 6 mesi. ${ }^{16}$

Al momento le evidenze disponibili a favore dell'utilizzo del Fe somministrato per via orale sono scarse e controverse. Il Fe orale sembra avere scarso effetto sui livelli di $\mathrm{Hb}$, sul metabolismo marziale o sui parametri cardiaci ${ }^{17}$ nei pazienti cardiopatici. Gli autori di un piccolo studio che includeva solo 18 pazienti ${ }^{18}$ non osservavano differenze tra $\mathrm{Fe}$ orale ed e.v. in termini di incremento dell'Hb, ma il picco di VO2 (massimo consumo di ossigeno) incrementa solo nel gruppo trattato con Fe e.v. Uno studio retrospettivo che includeva più di 100 pazienti affetti da $\mathrm{CHF}^{19}{ }^{19}$ ha dimostrato che il Fe per via orale migliora i depositi marziali e la disfunzione diastolica del ventricolo sinistro, nella stessa intensità descritta da altri autori che utilizzavano il farmaco per via endovenosa. Tuttavia, i risultati del recentissimo RCT intitolato IRONOUT $\mathrm{HF}^{20}$ mostrano chiaramente che nei pazienti con CHF a ridotta funzione sistolica (HFrEF) e ID, l'integrazione orale di $\mathrm{Fe}$ aumenta minimamente i depositi marziale ma non migliora la capacità di esercizio dopo 16 settimane di trattamento rispetto al gruppo placebo.

I risultati degli studi di integrazione di $\mathrm{Fe}$ per os presentano aspetti divergenti rispetto ai dati provenienti da altri studi di replezione di Fe per via e.v. ${ }^{21-23}$ Nonostante tutti gli studi siano stati eseguiti su popolazioni di caratteristiche simili, i risultati tendono a scartare l'uso dell'integrazione di Fe orale per il trattamento dell'ID in pazienti con HFrEF.

Tuttavia, prima di affrontare la scelta della via di somministrazione della terapia marziale nei pazienti con CHF con o senza anemia, sarebbe opportuno esaminare alcuni aspetti di non indifferente rilevanza:

$\checkmark$ Primo: poiché il trattamento dello CHF comporta l'uso di un regime farmacologico complesso e articolato $^{3}$ che mette a dura prova la compliance terapeutica da parte dei pazienti, l'alternativa di una terapia e.v. potrebbe essere più attraente, al fine anche di ridurre il numero di pillole assunte regolarmente.

$\checkmark$ Secondo: anche se la terapia con Fe e.v. e in particolare quella con FCM è economicamente più costosa rispetto all'integrazione di Fe orale, il rapporto costo-efficacia della terapia e.v. potrebbe essere valutata utilizzando altri parametri di misura come la qualità della vita e il minor numero di ospedalizzazioni. ${ }^{24}$

$\checkmark$ Terzo: di grande riguardo sono gli aspetti vincolati alla sicurezza, alla tolleranza della terapia. Se da un lato la terapia con Fe e.v. è più efficiente rispetto a quella con Fe orale, da l'altro lato presenta un maggior rischio di reazioni di ipersensibilità (HSR) tra cui la più temibile è l'anafilassi. ${ }^{25}$ Le HSR sono piuttosto rare ma possono essere fatali se non gestite tempestivamente. ${ }^{26}$ È interessante segnalare che tali reazioni erano più comuni con l'uso delle vecchie formulazioni di $\mathrm{Fe}$ destrano ad alto peso molecolare (HMW-ID) di quanto non lo siano oggi con i preparati più recenti di ultima generazione ${ }^{26,27}$ caratterizzati da pesi molecolari più bassi. La tabella 1 illustra i composti componenti di Fe attualmente disponibili sia per via orale (A) sia per via e.v. (B) con i rispettivi contenuti di ferro elemento. Per quanto riguarda i prodotti ad uso e.v. viene specificata l'eventuale necessità di eseguire un test previo alla somministrazione e la necessità di un tempo di osservazione dopo l'infusione.

$\checkmark$ Quarto: sebbene la terapia con Fe e.v sembra presentare dei vantaggi in termini di efficienza, non sono da trascurare gli aspetti logistici e/o organizzativi. A questo riguardo, una recente survey Lombarda ${ }^{28}$ condotta dopo la pubblicazione delle normative EMA del 2013 per la sicurezza dei composti contenente $\mathrm{Fe}$ ad uso e.v., ha evidenziato una notevole disparità nelle prescrizioni terapeutiche tra pazienti dializzati nei centri ospedalieri rispetto a quelli trattati nei centri periferici. L'adeguamento alla normativa ha generato variazioni strutturali e programmatiche nell'attività dei singoli centri ospedalieri. Questa osservazione mette in evidenza il ruolo rilevante degli schemi organizzativi delle diverse realtà sanitarie nel portare a termine $\mathrm{i}$ target proposti dalle linee guida. 
Tabella I. Contenuto di ferro elemento nei composti commercialmente disponibili per via orale (A) e per via endovenosa (B).

\begin{tabular}{lll}
\hline Molecola - Farmaco & Concentrazione di Ferro elemento & Necessità di osservazione \\
\hline A) Ferro orale & $120 \mathrm{mg} / \mathrm{g}$ & - \\
Pirofosfato ferrico & $120 \mathrm{mg} / \mathrm{g}$ & - \\
Gluconato ferroso & $200 \mathrm{mg} / \mathrm{g}$ & - \\
Solfato ferroso & $300 \mathrm{mg} / \mathrm{g}$ & - \\
Solfato ferroso, secco & $330 \mathrm{mg} / \mathrm{g}$ & - \\
Ferroso fumarato & $480 \mathrm{mg} / \mathrm{g}$ & - \\
Carbonato ferroso & $1000 \mathrm{mg} / \mathrm{g}$ & - \\
Ferro carbonile & & \\
B) Ferro endovenoso & $50 \mathrm{mg} / \mathrm{mL}$ & 60 min (dose di prova), \\
HMW-ID (DexFerrum) & & 30 min (dose effettiva) \\
LMW-ID (InFed) & $50 \mathrm{mg} / \mathrm{mL}$ & 60 min (dose di prova), \\
Gluconato ferrico (Ferlixit) & $12.5 \mathrm{mg} / \mathrm{mL}$ & 30 min (dose effettiva) \\
Ferro saccarosio (Venofer) & $20 \mathrm{mg} / \mathrm{mL}$ & 30 min \\
Ferumoxytol (Feraheme) & $30 \mathrm{mg} / \mathrm{mL}$ & 30 min \\
Ferro carbossimaltosio (Ferinject) & $50 \mathrm{mg} / \mathrm{mL}$ & 30 min \\
\hline
\end{tabular}

HMW-ID: high-molecular-weight iron dextran; LMW-ID: low-molecular-weight iron dextran.

Rispetto al Fe somministrato per via orale, il trattamento per via e.v. è stato studiato più a fondo nei pazienti con CHF. In uno studio relativamente piccolo e a breve termine con disegno randomizzato, controllato con placebo (FERRIC-HF), Okonko et al. ${ }^{23}$ dimostrano un miglioramento statisticamente e clinicamente significativo in termini di consumo di ossigeno, classe funzionale New York Heart Association (NYHA) e nella capacità di esercizio nei pazienti anemici e non-anemici con $\mathrm{CHF}$ trattati con Fe saccarosio.

Visti i promettenti risultati osservati nel FERRIC-HF trial, ${ }^{23}$ è stato progettato un altro studio clinico più ampio e più prolungato: il FAIR-HF trial, ${ }^{21}$ che aveva l'obbiettivo di valutare gli effetti del FCM sull'ID in pazienti con CHF non anemici. Il FAIR-HF trial ha arruolato 459 pazienti con CHF (Classe NYHA II o III, bassi livelli di ferritina). Gli autori di questo trial dimostrano un miglioramento nella classe funzionale NYHA, nella tolleranza all'esercizio e nella qualità di vita nei pazienti trattati con FCM. Inoltre, i tassi di mortalità, eventi avversi minori e gravi sono risultati simili rispetto al gruppo trattati con placebo.

Infine, il CONFIRM-HF Trial, $^{29}$ multi-centrico, in doppio cieco, controllato con placebo, è stato disegnato per valutare i vantaggi e la sicurezza di una terapia con $\mathrm{Fe}$ e.v. a lungo termine in pazienti con ID e CHF. Lo studio che arruolava 304 pazienti con $\mathrm{CHF}$ con $\mathrm{FE} \leqslant 45 \%$, aumento dei peptidi natriuretici e ID, dimostrava un significativo miglioramento del test del cammino nei pazienti trattati con FCM e.v. rispetto al placebo. Inoltre si osservava un miglioramento nella classe NYHA, negli score di qualità di vita e di fatica. Il trattamento con FCM veniva associato anche ad una significativa riduzione del rischio di ospedalizzazione per aggravamento della $\mathrm{CHF}$, mentre il numero di decessi e l'incidenza di eventi avversi sono risultati sovrapponibili tra i due gruppi.

I risultati di questi trial suggeriscono che in pazienti con CHF e in particolare in quelli con HFrEF, il trattamento con Fe somministrato per via orale può migliorare minimamente l'assetto marziale senza tuttavia fornire un significativo vantaggio sulla classe funzionale NYHA, sulla capacità di esercizio mediante il test del cammino di 6 minuti (6 min walk distance, 6MWD), sulla qualità di vita (QOL) e funzione cognitiva.

Nonostante le caratteristiche cliniche e biochimiche dei pazienti trattati con Fe orale nello studio IRONOUT HF ${ }^{20}$ fossero sovrapponibili a quelle dei due principali trial che utilizzavano Fe e.v. (FAIR-HF and CONFIRM-HF), i marker impiegati per caratterizzare il profilo marziali (sideremia, TIBC, Sat. Trasferrina e sTfR) erano significativamente migliori nei trattati con $\mathrm{Fe}$ e.v.

Malgrado sia stato somministrato circa 15 volte più $\mathrm{Fe}$ elemento per via orale nello studio IRONOUT HF ${ }^{20}$ rispetto alla quantità indicata e.v. nel FAIR-HF trial ${ }^{21}$ (in media: $33.6 \mathrm{~g}$ vs. $2 \mathrm{~g}$ di $\mathrm{Fe}$ ), è stato possibile osservare un modesto incremento del 3\% circa di TSAT e di $11 \mathrm{ng}$ di ferritina nei pazienti trattati per via orale rispetto ad un incremento del $70 \%$ di TSAT e di oltre il $500 \%$ di ferritina in quelli trattati per via e.v.

I risultati di una recente meta-analisi ${ }^{30}$ che includeva gli studi FAIR-HF trial $^{21}$ e CONFIRM-HF Trial, ${ }^{29}$ suggeriscono che il trattamento con FCM riduce le ospedalizzazioni associate al peggioramento della CHF o di qualsiasi ricovero per cause cardiovascolari con un utilizzo relativamente sicuro nel termine di 52 settimane di trattamento. Tuttavia, negli studi non emergevano benefici evidenti sulla mortalità, sebbene le stime fossero 
Tabella 2. Caratteristiche dei due principali trial sugli effetti del FCM del paziente con CHF.

\begin{tabular}{|c|c|c|}
\hline Caratteristiche degli studi & FAIR-HF'I & CONFIRM-HF' \\
\hline Numero totale di pazienti randomizzati & $\begin{array}{l}459 \\
\text { Braccio FMC: } 304 \\
\text { Braccio placebo: } 155\end{array}$ & $\begin{array}{l}304 \\
\text { Braccio FMC: I52 } \\
\text { Braccio placebo: } 152\end{array}$ \\
\hline Percentuale M/F per braccio (\%) & $\begin{array}{l}\text { Braccio FCM: } M=47.4 ; F=52.3 \% \\
\text { Braccio placebo: } M=45.2 ; F=54.8 \%\end{array}$ & $\begin{array}{l}\text { Braccio FCM: } M=55 ; F=45 \% \\
\text { Braccio placebo: } M=51 ; F=49 \%\end{array}$ \\
\hline Età media (anni) & $\begin{array}{l}\text { Braccio FCM: } 67.8 \pm 10.3 \\
\text { Placebo: } 67.4 \pm 11.1\end{array}$ & $\begin{array}{l}\text { Braccio FCM: } 68.8 \pm 9.5 \\
\text { Placebo: } 69.5 \pm 9.3\end{array}$ \\
\hline Farmaco e dose utilizzati & FCM e.v. (200 mg settimanali) & FCM e.v. (500-1000 mg settimanali) \\
\hline Placebo & Soluzione Salina & Soluzione Salina \\
\hline Durata del Follow-up & 26 settimane & 52 settimane \\
\hline \multicolumn{3}{|l|}{ Criteri di inclusione } \\
\hline \multicolumn{3}{|l|}{$\checkmark$ Criteri ematologici } \\
\hline - $\quad$ TSAT $(\%)$ & $<20 \%$ & $<20 \%$ \\
\hline - Ferritin $(\mathrm{ng} / \mathrm{mL})$ & $<100$ (or 100-299 se TSAT <20\%) & $<100$ (or $100-300$ se TSAT $<20 \%$ ) \\
\hline - $\mathrm{Hb}(\mathrm{gm} / \mathrm{dL})$ & 9.5 to 13.5 & $<15$ \\
\hline \multicolumn{3}{|l|}{$\checkmark$ Criteri cardiaci } \\
\hline$-\quad \operatorname{LVEF}(\%)$ & $<40 \%$ con NYHA II, $<45 \%$ con NYHA III & $\leqslant 45 \%$ \\
\hline - NYHA class & dalla II alla III & dalla II alla III \\
\hline - BNP e/o Nt-pro-BNP & - & $>100 \mathrm{pg} / \mathrm{mL}$ e/ o $>400 \mathrm{pg} / \mathrm{mL}$ \\
\hline \multicolumn{3}{|l|}{$\checkmark \quad$ Criteri nefrologici } \\
\hline - $\operatorname{eGFR}\left(\mathrm{ml} / \mathrm{min} / \mathrm{l} .73 \mathrm{~m}^{2}\right)$ & $\begin{array}{l}\text { Braccio FCM: } 63.8 \pm 21.2 \\
\text { Placebo: } 64.8 \pm 25.3\end{array}$ & $\begin{array}{l}\text { Braccio FCM: } 66.4 \pm 21.7 \\
\text { Placebo: } 63.5 \pm 20.9\end{array}$ \\
\hline
\end{tabular}

FCM: ferro carbossimaltosio, TSAT: saturazione della trasferrina (\%), Hb: emoglobina, NYHA: New York Heart Association, LVEF: left ventricular ejection fraction, BNP: brain natriuretic peptide, Nt-Pro-BNP: N-terminal-pro-brain natriuretic peptide, eGFR: estimated glomerular filtration rate.

leggermente a favore di FCM rispetto al placebo. Questo potrebbe essere dovuto a un numero molto basso di decessi su entrambi gli studi. La tabella numero 2 riassume le caratteristiche cliniche dei due principali trial sull'uso del FCM sui pazienti con CHF.

\section{La terapia con ESA nell'anemia cardio-renale}

Molti piccoli studi hanno verificato gli effetti dell'aumento dell'Hb in pazienti con CHF mediante l'uso di ESA. I pazienti inclusi in questi studi hanno eseguito un trattamento sia con ESA da sola sia con l'integrazione di Fe orale o e.v.

Silverberg et al. ${ }^{31}$ sono stati tra i primi autori a valutare la prevalenza e la severità dell'anemia in pazienti con $\mathrm{CHF}$ e gli effetti della sua correzione sugli esiti di tipo renale e cardiovascolare. Un gruppo di 26 pazienti cardiopatici che avevano eseguito una terapia massimale per lo scompenso congestizio per almeno 6 mesi, sono stati successivamente trattati con eritropoietina alfa per via sottocutanea (s.c.) e Fe per via e.v. per altri 7 mesi, con l'obiettivo di incrementare i valori di $\mathrm{Hb}$ a $12 \mathrm{~g} / \%$. Gli autori osservavano che la prevalenza dell'anemia aumentava con la severità dello CHF. Inoltre, l'aumento dell'Hb ottenuto con il trattamento comportava un miglioramento della classe funzionale NYHA e della funzione sistolica (left ventricular ejection fraction, $\mathrm{LVEF} \%$ ). Infine, gli autori osservavano che dopo la correzione dell'Hb, l'ospedalizzazione si riduceva di quasi il $92 \%$ rispetto al periodo precedente al trattamento. Risultati analoghi sono stati riportati dagli stessi autori in un altro studio RCT in cui il trattamento con ESA comportava una riduzione nell'ospedalizzazione dovuta ad aggravamento della classe NYHA.

Anche altri autori ${ }^{32}$ hanno mostrato risultati similari. Dopo 3 mesi di trattamento con ESA, Fe orale e folati, Mancini et al. dimostravano un incremento dei livelli di $\mathrm{Hb}$, del 6MWD e del picco di $\mathrm{VO}_{2}$ nel gruppo trattati rispetto a braccio placebo. Più recentemente, uno studio $\mathrm{RCT}^{33}$ che includeva 51 pazienti anemici con CHF ha riportato che dopo 1 anno di trattamento con eritropoietina beta e Fe orale si osservava una riduzione del volume e della massa ventricolare sinistra, un miglioramento della $\mathrm{LVEF} \%$ e delle pressioni polmonari. Inoltre, rispetto ai controlli, i pazienti trattati riducevano sensibilmente la necessità di ospedalizzazione per cause cardiovascolari.

Nello studio RCT di Ponikowski ${ }^{34}$ che includeva 41 pazienti anemici con $\mathrm{CHF}$, il trattamento con darbepoietina alfa determinava un incremento sensibile dei livelli di $\mathrm{Hb}$ e un miglioramento della qualità di vita. Invece, gli autori non riuscivano a dimostrare differenze significative per quanto riguarda il picco di $\mathrm{VO}_{2}$, la capacità all'esercizio (6MWD), i valori di BNP e la funzione renale.

In un altro singolo studio RCT, in doppio cieco, ${ }^{35}$ controllato con placebo su 165 pazienti con $\mathrm{CHF}$ ipocinetica $(\mathrm{LVEF}=40 \%)$ e anemia trattati con darbepoetin alfa, non emergevano differenze significative tra i pazienti 
Tabella 3. Principali trial randomizzati controllati sul trattamento dell'anemia con ESA in cardiopatici cronici.

\begin{tabular}{|c|c|c|c|c|c|c|}
\hline Autore & $\mathrm{N}$ & $\begin{array}{l}\text { Disegno Caso/ } \\
\text { controllo }\end{array}$ & $\begin{array}{l}\text { Altri } \\
\text { farmaci }\end{array}$ & $\begin{array}{l}\text { F -up } \\
\text { (mesi) }\end{array}$ & End-point & Conclusioni \\
\hline Silverberg ${ }^{31}$ & 26 & Epoietin alfa & Ferro & 7 & $\begin{array}{l}\text { Classe NHYA, LVEF, } \\
\text { ospedalizzazione, tolleranza } \\
\text { fisica }\end{array}$ & $\begin{array}{l}\text { L'incremento dell'Hb si associa al } \\
\text { miglioramento della classe NHYA e } \\
\text { LVEF, e alla riduzione del diuretico e } \\
\text { dell'ospedalizzazione }\end{array}$ \\
\hline Mancini ${ }^{32}$ & 23 & $\begin{array}{l}\text { Epoietin alfa } \\
\text { vs placebo }\end{array}$ & Ferro & 3 & $\begin{array}{l}\text { Capacità all'esercizio, } \\
\text { funzione vasodilatatoria }\end{array}$ & $\begin{array}{l}\text { La terapia con EPO comporta un } \\
\text { incremento significativo dell'Hb, } \\
\text { incremento del } 6 M W D \text { e del picco di } \\
\text { VO2 }\end{array}$ \\
\hline Palazzuoli33 & 51 & $\begin{array}{l}\text { Epoietin beta } \\
\text { vs placebo }\end{array}$ & Ferro & 12 & $\begin{array}{l}\text { Primari: Dimensioni del LV, } \\
\text { LVEF, Hb, Cr, BNP } \\
\text { Secondari: eventi CV, peso, } \\
\text { edema NYHA }\end{array}$ & $\begin{array}{l}\text { Nel gruppo trattato aumenta l'Hb e } \\
\text { la LVEF, e diminuiscono: dimensioni e } \\
\text { volume della LVM, PAPs, BNP e necessità } \\
\text { di ricovero, mentre non si osservano } \\
\text { cambiamenti nel gruppo di controllo. }\end{array}$ \\
\hline Ponikowski ${ }^{34}$ & $4 I$ & $\begin{array}{l}\text { Darbepoietin alfa } \\
\text { vs placebo }\end{array}$ & Ferro & 6 & $\begin{array}{l}\text { Primari: capacità all'esercizio } \\
\text { Secondari: picco VO2, } \\
\text { tenuta fisica, Hb, Classe } \\
\text { NYHA, QOL, BNP, peso, } \\
\text { ospedalizzazione }\end{array}$ & $\begin{array}{l}\text { Nessuna differenza è stata rilevata per } \\
\text { quanto riguarda picco VO2, durata } \\
\text { dell'esercizio, BNP e funzione renale tra i } \\
\text { gruppi di trattamento. }\end{array}$ \\
\hline $\begin{array}{l}\text { van } \\
\text { Veldhuisen }{ }^{35}\end{array}$ & 165 & $\begin{array}{l}\text { Darbepoietin alfa } \\
\text { vs placebo }\end{array}$ & Ferro & 6 & $\begin{array}{l}\text { Primari: Tasso di incremento } \\
\text { dell'Hb } \\
\text { Secondari: cambi nella LVEF, } \\
\text { 6MWD, NYHA, QOL, PGA }\end{array}$ & $\begin{array}{l}\text { Incremento dell'Hb equivalente tra } \\
\text { i gruppi. Rilevati miglioramenti non } \\
\text { significativi per 6MWD nel gruppo } \\
\text { trattato }\end{array}$ \\
\hline Ghali ${ }^{36}$ & 319 & $\begin{array}{l}\text { Darbepoietin alfa } \\
\text { vs placebo }\end{array}$ & Ferro & 12 & $\begin{array}{l}\text { Primari: capacità all'esercizio } \\
\text { Secondari: NYHA, QOL, } \\
\text { mortalità, ospedalizzazione }\end{array}$ & $\begin{array}{l}\text { Il trattamento con EPO non ha migliorato } \\
\text { durata dell'esercizio, classe NYHA o } \\
\text { QOL rispetto al placebo. Incremento } \\
\text { dell'Hb equivalente tra i gruppi. Rilevati } \\
\text { miglioramenti per 6MWD nel gruppo } \\
\text { trattato. }\end{array}$ \\
\hline Swedberg ${ }^{37}$ & 2278 & $\begin{array}{l}\text { Darbepoietin alfa } \\
\text { vs placebo }\end{array}$ & Ferro & 28 & $\begin{array}{l}\text { Primari: tutte le cause di } \\
\text { mortalità o primo ricovero } \\
\text { per scompenso } \\
\text { Secondari: mortalità } \\
\mathrm{CV} \text {, primo episodio di } \\
\text { scompenso, MI, ictus, } \\
\text { ospedalizzazione, SCD, QOL }\end{array}$ & $\begin{array}{l}\text { Non differenze tra i gruppi per l'outcome } \\
\text { primario. Nei pazienti trattati aumenta } \\
\text { del tasso di eventi tromboembolici. }\end{array}$ \\
\hline
\end{tabular}

6MWD: test del cammino di 6 minuti, BNP: peptide natriuretico cerebrale, Cr: creatinina, CV: cardiovascolare, ESA: agente stimolante l'eritropoiesi, $\mathrm{Hb}$ : emoglobina, CHF: insufficienza cardiaca congestizia, LV: ventricolo sinistro, LVEF: frazione di eiezione ventricolare sinistra, MI: infarto miocardico, NYHA: New York Heart Association, QOL: qualità della vita, SCD: morte cardiaca improvvisa, VO2: volume di ossigeno, PGA: punteggio di valutazione globale del paziente.

trattati rispetto a e quelli nel braccio placebo per quanto riguarda il 6MWD e il punteggio di valutazione globale del paziente. Non emergevano neanche sensibili differenze per gli stessi parametri all'interno del gruppo dei trattati con ESA, ossia: tra quelli a dosaggio fisso $(50 \mu \mathrm{g})$ e quelli a dosaggio aggiustato per il peso $(0,75 \mu \mathrm{g} / \mathrm{kg})$.

Data la scarsa campionaria e l'assenza di un disegno sperimentale a doppio cieco di questi trial, gli effetti benefici documentati non possono essere applicati su vasta scala. Inoltre, pochi di questi studi hanno valutato gli esiti più rilevanti come cause di morte e di ospedalizzazione.

Nei due più ampi RCT multicentrici in doppio cieco controllati con placebo che hanno utilizzato Darbepoetin: STAMINA-HF ${ }^{36}$ e RED-HF, ${ }^{37}$ non si sono verificati miglioramenti significativi in termini di classe funzionale NYHA, morbidità (espressa come ospedalizzazione) o mortalità (per tutte le cause).

Nello studio STAMINA-HF, 36319 pazienti con CHF e LVEF $<40 \%$ sono stati randomizzati a placebo o darbepoetin alfa per 1 anno. Nonostante l'aumento dei livelli di $\mathrm{Hb}$ nei pazienti trattati, non si è verificato un migliorato significativo della tolleranza all'esercizio, della classe NYHA o della qualità della vita rispetto al placebo. Il braccio dei trattati mostrava una differenza non significativa verso un minor rischio di mortalità per tutte le cause e la prima ospedalizzazione per cause cardiovascolari rispetto al placebo. L'incidenza di altri eventi avversi incluso lo sviluppo di ipertensione e infarto del miocardio è stata simile nei due gruppi. 
Nel più grande RCT multicentrico controllato RED-HF, ${ }^{37}$ che arruolava 2.278 pazienti con CHF sistolico (LVEF < $40 \%$, classe NYHA $>2$ ) e anemia $(\mathrm{Hb} 9-12 \mathrm{~g} / \mathrm{dL})$, la dose $\overline{\mathrm{di}}$ darbepoetin alfa è stata aggiustata per portare a un target di $\mathrm{Hb}>$ a $13 \mathrm{~g} / \mathrm{dL}$. Dopo un follow-up mediano di 28 mesi, $l^{\prime} \mathrm{Hb}$ è aumentata nel braccio attivo (da 11,2 a 13,0 g/dL), rimanendo invariata nel gruppo placebo. Non sono emerse differenze significative tra i trattati e i controlli per l'esito primario composito di morte per qualsiasi causa o primo ricovero ospedaliero. Pertanto, nessun beneficio è stato riscontrato rispetto a tutti gli altri esiti secondari tra cui mortalità per tutte le cause, morte cardiovascolare, ospedalizzazione correlata a CHF ed eventi coronarici.

Una recentissima meta-analisi ${ }^{38}$ che includeva 3044 pazienti provenienti da 11 studi ha documentato che il trattamento con ESA comporta un aumento significativo di livelli di $\mathrm{Hb}$, della $\mathrm{LVEF} \%$, una netta riduzione del BNP e della necessità di ospedalizzazione, e un miglioramento della classe funzionale NHYA. Tuttavia, non si evidenziano differenze significative tra i pazienti trattati con ESA e quelli non trattati in termini di mortalità per tutte le cause. Infatti, gli autori concludono che sebbene il trattamento dell'anemia con ESA in pazienti con CHF non riduce il tasso di mortalità, la terapia può contribuire al miglioramento sintomatico dei pazienti. La tabella 3 espone $\mathrm{i}$ principali trial randomizzati controllati sul trattamento con ESA nei pazienti con CHF.

\section{Conclusioni}

L'anemia è una condizione clinica comunemente associata a CRS, ed è correlata ad esiti sfavorevoli come ospedalizzazione e aumento della mortalità. Le attuali linee guida raccomandano un adeguato percorso diagnostico e la ricerca sistematica dell'ID in tutti i pazienti con CRAS.

L'ID ferro è altamente prevalente nei pazienti con CHF ed è associata indipendentemente dalla presenza o assenza di anemia, a compromissione della qualità della vita, ridotta capacità di esercizio e aumento della mortalità. Esistono due opzioni di trattamento per l'ID: la terapia con Fe orale e quella e.v. Le evidenze riguardanti l'utilizzo del Fe orale sono controverse. La maggior parte degli studi suggeriscono che il $\mathrm{Fe}$ orale ha uno scarso effetto sui livelli di $\mathrm{Hb}$, sul metabolismo marziale o sui parametri cardiovascolari. Diversamente, l'integrazione di $\mathrm{Fe}$ e.v. induce un miglioramento dei sintomi, della capacità funzionale e della qualità della vita, e può ridurre il rischio di ospedalizzazione connesso al peggioramento del CHF e alla riduzione della LVEF. Bisognerà attendere il risultato dei nuovi trial randomizzati volti a valutare gli esiti clinici a lungo termine.

L'uso degli ESA nel trattamento dell'anemia della CRS è attualmente molto dibattuto. Molti autori confermano la riduzione del BNP, il miglioramento della funzione sistolica ventricolare (LVEF\%) e della classe funzionale NYHA. Allo stesso tempo, la terapia con ESA sembra aumentare la tolleranza all'esercizio. Tuttavia, non è stato possibile dimostrare una riduzione del tasso di mortalità.

Di conseguenza, il trattamento dell'anemia con l'ESA migliora i sintomi associati alla $\mathrm{CHF}$, ma non ha migliorato gli esiti clinici.

\section{Dichiarazione di assenza di conflitto di interessi}

Gli Autori dichiarano di non avere conflitti di interessi.

\section{Finanziamenti}

Gli Autori dichiarano di non aver ricevuto finanziamenti specifici da qualsiasi ente nei settori pubblico, private o senza fini di lucro.

\section{Bibliografia}

1. Rivera RF, Sciarrone Alibrandi MT, Di Lullo L, Floccari F, De Pascalis A, Bellasi A and Ronco C. The cardiorenal anemia syndrome. Part one: epidemiology and clinical aspects. G Tec Nefrol E Dialitiche 2017; 29: 157-232.

2. Ronco C, McCullough P, Anker SD, Anand I, Aspromonte $\mathrm{N}$, Bagshaw SM, et al. Cardio-renal syndromes: report from the consensus conference of the acute dialysis quality initiative. Eur Heart J 2010; 31: 703-711.

3. Ponikowski P, Voors AA, Ander SD, et al. 2016 ESC Guidelines for the diagnosis and treatment of acute and chronic heart failure: The Task Force for the diagnosis and treatment of acute and chronic heart failure of the European Society of Cardiology (ESC). Developed with the special contribution of the Heart Failure Association (HFA) of the ESC. treatment of acute and chronic heart failure of the European Society of Cardiology (ESC). Developed with the special contribution of the Heart Failure Association (HFA) of the ESC. Eur J Heart Fail 2016; 18: 891-975.

4. KDOQI Clinical Practice Guideline and Clinical Practice Recommendations for anemia in chronic kidney disease: 2007 update of hemoglobin target. Am J Kidney Dis 2007; 50: 471-530.

5. Chapter 1: Diagnosis and evaluation of anemia in CKD. Kidney Int Suppl 2012; 2: 288-291.

6. US Renal Data System: USRDS 2011 Annual Data Report: Atlas of Chronic Kidney Disease and End-Stage Renal Disease in the United States. Bethesda, MD: National Institutes of Health, National Institute of Diabetes and Digestive and Kidney Diseases, 2011.

7. Practice Guidelines for Blood Component Therapy: A report by the American Society of Anesthesiologists Task Force on Blood Component Therapy. Anesthesiology 1996; 84: 732-747.

8. Brownlie T, Utermohlen V, Hinton PS, et al. Marginal iron deficiency without anemia impairs aerobic adaptation among previously untrained women. Am J Clin Nutr 2002; 75: 734-742.

9. Brownlie T, Utermohlen V, Hinton PS, et al. Tissue iron deficiency without anemia impairs adaptation in endurance capacity after aerobic training in previously untrained women. Am J Clin Nutr 2004; 79: 437-443.

10. Favrat B, Balck K, Breymann C, et al. Evaluation of a single dose of ferric carboxymaltose in fatigued, iron-deficient women-PREFER a randomized, placebo-controlled study. PloS One 2014; 9: e94217. 
11. Klip IT, Comin-Colet J, Voors AA, et al. Iron deficiency in chronic heart failure: an international pooled analysis. $\mathrm{Am}$ Heart J 2013; 165: 575-582.e3.

12. Okonko DO, Mandal AKJ, Missouris CG, et al. Disordered iron homeostasis in chronic heart failure: prevalence, predictors, and relation to anemia, exercise capacity, and survival. J Am Coll Cardiol 2011; 58: 1241-1251.

13. Wienbergen $\mathrm{H}$, Pfister $\mathrm{O}$, Hochadel $\mathrm{M}$, et al. Usefulness of Iron Deficiency Correction in Management of Patients With Heart Failure [from the Registry Analysis of Iron Deficiency-Heart Failure (RAID-HF) Registry]. Am $J$ Cardiol 2016; 118: 1875-1880.

14. Rivera RF, Alibrandi MTS, Di Lullo L, et al. Clinical management of anemia in patients with CKD. G Ital Nefrol 2017; 34 (Suppl 69): 20-35.

15. Rivera RF, Di Lullo L, De Pascalis A, et al. anemia in patients with chronic kidney disease: current screening and management approaches. Nephrol Ren Dis 2016; 1: 1-9.

16. Filippatos G, Farmakis D, Colet JC, et al. Intravenous ferric carboxymaltose in iron-deficient chronic heart failure patients with and without anaemia: a subanalysis of the FAIR-HF trial. Eur J Heart Fail 2013; 15: 1267-1276.

17. McDonagh $\mathrm{T}$ and Macdougall IC. Iron therapy for the treatment of iron deficiency in chronic heart failure: intravenous or oral? Eur J Heart Fail 2015; 17: 248-262.

18. Beck-da-Silva L, Piardi D, Soder S, Rohde LE, PereiraBarretto AC, de Albuquerque D, et al. IRON-HF study: a randomized trial to assess the effects of iron in heart failure patients with anemia. Int J Cardiol 2013; 168: 3439-3442.

19. Niehaus ED, Malhotra R, Cocca-Spofford D, et al. Repletion of Iron Stores With the Use of Oral Iron Supplementation in Patients With Systolic Heart Failure. J Card Fail 2015; 21: 694-697.

20. Lewis GD, Malhotra R, Hernandez AF, et al. Effect of Oral Iron Repletion on Exercise Capacity in Patients With Heart Failure With Reduced Ejection Fraction and Iron Deficiency: The IRONOUT HF Randomized Clinical Trial. JAMA 2017; 317: 1958-1966.

21. Anker SD, Kirwan B-A, van Veldhuisen DJ, et al. Effects of ferric carboxymaltose on hospitalisations and mortality rates in iron-deficient heart failure patients: an individual patient data meta-analysis. Eur J Heart Fail 2018; 20: 125-133.

22. Ponikowski P, van Veldhuisen DJ, Comin-Colet J, et al. Beneficial effects of long-term intravenous iron therapy with ferric carboxymaltose in patients with symptomatic heart failure and iron deficiency $\dagger$. Eur Heart $J$ 2015; 36: $657-668$

23. Okonko DO, Grzeslo A, Witkowski T, et al. Effect of intravenous iron sucrose on exercise tolerance in anemic and nonanemic patients with symptomatic chronic heart failure and iron deficiency FERRIC-HF: a randomized, controlled, observer-blinded trial. J Am Coll Cardiol 2008; 51: 103-112.

24. Hofmarcher $\mathrm{T}$ and Borg S. Cost-effectiveness analysis of ferric carboxymaltose in iron-deficient patients with chronic heart failure in Sweden. J Med Econ 2015; 18: 492-501.

25. Rampton D, Folkersen J, Fishbane S, et al. Hypersensitivity reactions to intravenous iron: guidance for risk minimization and management. Haematologica 2014; 99: 1671-1676.
26. Bircher AJ and Auerbach M. Hypersensitivity from intravenous iron products. Immunol Allergy Clin North Am 2014; 34: 707-723, x-xi.

27. Auerbach M, Adamson J, Bircher A, Breymann C, Fishbane $\mathrm{S}$, Gafter-Gvili A, et al. On the safety of intravenous iron, evidence trumps conjecture. Haematologica 2015; 100: e214-215.

28. Rivera RF, Guido D, Del Vecchio L, et al. Impact of European medicines agency recommendations for hypersensitivity reactions on intravenous iron prescription in haemodialysis centres of the Lombardy region. J Nephrol 2016; 29: 673-681.

29. Ponikowski P, van Veldhuisen DJ, Comin-Colet J, et al. Rationale and design of the CONFIRM-HF study: a doubleblind, randomized, placebo-controlled study to assess the effects of intravenous ferric carboxymaltose on functional capacity in patients with chronic heart failure and iron deficiency. ESC Heart Fail 2014; 1: 52-58.

30. Dalal J, Katekhaye V and Jain R. Effect of ferric carboxymaltose on hospitalization and mortality outcomes in chronic heart failure: A meta-analysis. Indian Heart $J$ 2017; 69: 736-741.

31. Silverberg DS, Wexler D, Blum M, et al. The use of subcutaneous erythropoietin and intravenous iron for the treatment of the anemia of severe, resistant congestive heart failure improves cardiac and renal function and functional cardiac class, and markedly reduces hospitalizations. $J \mathrm{Am}$ Coll Cardiol 2000; 35: 1737-1744.

32. Mancini DM, Katz SD, Lang CC, et al. Effect of erythropoietin on exercise capacity in patients with moderate to severe chronic heart failure. Circulation 2003; 107: 294-299.

33. Palazzuoli A, Silverberg DS, Iovine F, et al. Effects of betaerythropoietin treatment on left ventricular remodeling, systolic function, and B-type natriuretic peptide levels in patients with the cardiorenal anemia syndrome. Am Heart $J$ 2007; 154: 645.e9-15.

34. Ponikowski P, Anker SD, Szachniewicz J, et al. Effect of darbepoetin alfa on exercise tolerance in anemic patients with symptomatic chronic heart failure: a randomized, double-blind, placebo-controlled trial. J Am Coll Cardiol 2007; 49: 753-762.

35. van Veldhuisen DJ, Dickstein K, Cohen-Solal A, et al. Randomized, double-blind, placebo-controlled study to evaluate the effect of two dosing regimens of darbepoetin alfa in patients with heart failure and anaemia. Eur Heart $J$ 2007; 28: 2208-2216.

36. Ghali JK, Anand IS, Abraham WT, et al. Randomized double-blind trial of darbepoetin alfa in patients with symptomatic heart failure and anemia. Circulation 2008; 117: 526-535.

37. Swedberg K, Young JB, Anand IS, et al. Treatment of anemia with darbepoetin alfa in systolic heart failure. $N$ Engl J Med 2013; 368: 1210-1219.

38. Zhang H, Zhang P, Zhang Y, et al. Effects of erythropoiesisstimulating agents on heart failure patients with anemia: a meta-analysis. Postepy Kardiol Interwencyjnej 2016; 12: 247-253. 